\title{
PARALLEL VECTOR FIELDS AND PERIODIC ORBITS
}

\author{
SOL SCHWARTZMAN
}

\begin{abstract}
Let $V$ be a parallel vector field on a compact Riemannian manifold without boundary. Suppose the Euler class over the reals of the normal bundle to $V$ is different from zero. Then the flow defined by $V$ has a periodic orbit.
\end{abstract}

Let $M^{n}$ be a $C^{\infty}$ compact oriented $n$-dimensional Riemannian manifold, and let $V$ be a nowhere vanishing $C^{\infty}$ contravariant vector field on $M^{n}$ that is parallel with respect to the metric; that is, we assume that the covariant derivative of $V$ is zero. Let $\mu \in H^{n-1}\left(M^{n}, R\right)$ be the Euler class of the normal bundle to $V$ with real coefficients, where we put the obvious orientation on the normal bundle. The purpose of the note is to prove the following result:

THEOREM. If $\mu \neq 0$, the flow defined by $V$ has a periodic orbit.

PRoOF. We proceed by adopting a device used in [2]. The one-form $\alpha$ gotten from $V$ by lowering indices has covariant derivative zero and therefore has exterior derivative zero, since the exterior derivative is the skewsymmetrization of the covariant derivative. Moreover the interior product of $\alpha$ and $V$ is certainly never zero. Let $\alpha_{1}, \cdots, \alpha_{k}$ be closed one-forms on $M^{n}$ corresponding to a basis for the one-dimensional cohomology on $M^{n}$. For some $\delta>0,\left|\varepsilon_{1}\right|+\cdots+\left|\varepsilon_{k}\right|<\delta$ implies that $\alpha+\varepsilon_{1} \alpha_{1}+\cdots+\varepsilon_{k} \alpha_{k}$ has a nowhere vanishing interior product with $V$. We see from this that we can get $C^{\infty}$ one-forms $\omega_{1}, \cdots, \omega_{k}$ each of which has a nonvanishing interior product with $V$ and which determine cohomology classes which arise from a basis for the rational one-dimensional cohomology of $M^{n}$. Then by multiplying $\omega_{1}, \cdots, \omega_{k}$ by suitable rational constants we can get one-forms $\omega_{1}^{\prime}, \cdots, \omega_{k}^{\prime}$ each of which has a nowhere vanishing interior product with $V$ and which correspond to a basis of $H^{\prime}\left(M^{n}, R\right)$ arising from the integral one-dimensional cohomology of $M^{n}$. Each of the oneforms $\omega_{1}^{\prime}, \cdots, \omega_{k}^{\prime}$ arises from a map to the circle; in an easily understood notation there exist functions $\theta_{1}, \cdots, \theta_{k}$ on $M^{n}$ defined mod 1 such that $\omega_{1}^{\prime}=d \theta_{1}, \cdots, \omega_{k}^{\prime}=d \theta_{k}$.

Next we observe that if $N_{1}, \cdots, N_{k}$ are the $(n-1)$-dimensional manifolds corresponding to the equations $\theta_{1}=0, \cdots, \theta_{k}=0$ and taken with

Received by the editors November 22, 1972 and, in revised form, August 30, 1973. AMS (MOS) subject classifications (1970). Primary 34C40, 34C25.

Key words and phrases. Periodic orbit, parallel vector field, Euler class, flow.

(c) American Mathematical Society 1974 
the obvious orientation the fundamental class of $N_{i}$ yields by injection the element of $H_{n-1}\left(M^{n}, R\right)$ which corresponds by Poincaré duality to the cohomology class determined by $\omega_{i}^{\prime}$. This can be seen, for example, by noticing that for any closed $(n-1)$-form $\lambda, \int_{M_{n}} \lambda \wedge d \theta_{i}=\int_{N_{i}} \lambda$, which follows by using the local product structure on $M^{n}$ as a bundle over the circle and noting that the integral of $\lambda$ is the same over each fibre for any one of our fibrations $\theta_{i}$.

Since the Euler class $\mu$ of the normal bundle to the vector field $V$ is assumed different from zero, there is an $i_{0}$ such that the cap product of $\mu$ with the injection of the fundamental class of $N_{i_{0}}$ into the homology of $M^{n}$ is different from zero. Then the pullback of $\mu$ to the cohomology of $N_{i_{0}}$ is different from zero. This pullback is, however, just the Euler class of the oriented tangent bundle to $N_{i_{0}}$; thus the Euler characteristic of $N_{i_{0}}$ is different from zero.

However $N_{i_{0}}$ is clearly a global cross-section to the flow determined by the vector field $V$. If $h$ is the homeomorphism of $N_{i_{0}}$ onto itself determined by the flow we can conclude by a theorem of Fuller, since the Euler characteristic of $N_{i_{0}}$ is different from zero, that there exists a point on $N_{i_{0}}$ periodic under $h$. Then the orbit of this point under the flow defined by $V$ must be periodic.

(Note. After this paper was submitted, two related papers came to the author's attention. In [2] Conley introduced the notion of a flow which carries a one-form. A flow defined by a parallel vector field on a Riemannian manifold carries a closed one-form. Moreover if the Euler class over the reals of the normal bundle to a vector field $V$ on a compact orientable manifold is different from zero, and if the flow defined by $V$ carries a closed one-form, our argument can be carried over to prove that there is a periodic orbit. In [1], which appeared after the present paper was accepted for publication, Churchill shows that a flow which carries a closed one-form has a cross-section.)

\section{BiBLIOGRAPHY}

1. Richard C. Churchill, Invariant sets which carry cohomology, J. Differential Equations 13 (1973), 523-550.

2. C. Conley, Invariant sets which carry a one form, J. Differential Equations 8 (1970), 587-594. MR 43 \#5122.

3. F. Brock Fuller, The existence of periodic points, Ann. of Math. (2) 57 (1953), 229-230. MR 14, 669.

4. D. Tischler, On fibering certain foliated manifolds over $S^{1}$, Topology 9 (1970), 153-154. MR 41 \#1069.

Department of Mathematics, University of Rhode Island, Kingston, Rhode ISLAND 02881 